\title{
Widely-expressed and conserved long noncoding RNAs LINC00493 and LINC01420 influence on cell physiology
}

\author{
D.O. Konina ${ }^{1}$, P.A. Sparber ${ }^{2}$, A.Yu. Filatova 2 , I.A. Krivosheeva ${ }^{2}$, M.Yu. Skoblov ${ }^{1,2,3}$ \\ ${ }^{1}$ Department of Biological and Medical Physics, Moscow Institute of Physics and Technology \\ (State University), Dolgoprudny, Moscow region, Russia \\ ${ }^{2}$ Research Centre of Medical Genetics, Moscow, Russia \\ ${ }^{3}$ Far Eastern Federal University, Vladivostok, Russia \\ *e-mail: darya.konina@phystech.edu
}

Key words: Long noncoding RNA, RACE, MTT assay

Motivation and Aim: According to the date of GENCODE project (v 24), 15,767 long noncoding RNA (lncRNA) genes were implicated in humans. But functions have been revealed only for a small part of them. Long noncoding RNAs can act as regulators at many different levels of gene expression, including chromatin organization, transcriptional regulation and posttranscriptional control. Moreover, lncRNAs play important roles in disease. To date, it has been confirmed that there are about 3000 lncRNA-disease entries in accordance with lncRNADisease database. Improving our knowledge about lncRNAs will lead to more complete understanding of the mechanisms of biological processes at the molecular level in the body and help to optimize and systematize the diagnosis of hereditary human diseases, as well as develop approaches for their therapy. This work is devoted to the determination of the structures and functions of the new long non-coding RNAs LINC01420 and LINC00493. Methods: Nucleotide sequences of the studied genes were found in following databases: RefSeq, Ensemble, GENCODE. Conservation and expression level in various human cell lines and tissues were analyzed using data from the UCSC genomic browser. The reverse transcription reaction, PCR, RACE-PCR was used to determine the structures of the RNAs. To study the expression profile, RT-q PCR was performed on RNA samples from various human cell lines. To define functions of genes, we investigated its subcellular localization by using soft lysis and analyzing cytoplasmic compartment, nucleoplasm and chromatin fraction of RNA. In addition, we performed effective knockdown of lncRNAs using lipofection with 2 siRNAs for each gene in A375 cell line. Then, we observed the variation of cell proliferation by MTT assay. To study cell migration in vitro wound-healing assay was performed.

Results: Bioinformatic analysis showed that these LINCs are highly expressed in many human cell lines and tissues. The profile of the distribution of LINCs expression in human tissues allows to attribute these genes to the group of "housekeeping genes". RT-PCR analysis revealed that LINC01420 has 3 exons and 2 isoforms: short-major, long-minor. LINC00493 has 3 exons and 1 main isoform. We determined the exact $5^{\prime}$ and $3^{\prime}$-ends of the studied RNAs by RACE. We established that the transcripts of LINCs have cytoplasmic localization. Besides, we performed LINCs knockdown experiments followed by proliferation and migration assays. Results of MTT-assay indicated that the cell proliferation was attenuated after LINC00493 knockdown, while downregulation of LINC01420 didn't affect cell viability. On the other hand, wound healing assay showed that the migration ability was decreased with knockdown of studied genes.

Conclusion: In our study, the exact structure of two lncRNAs was obtained. We revealed main LINC's isoforms and number of exons. We also received data on their subcellular localization, the effect on viability and migration of cells. Thus, we have come closer to understanding function and mechanism of action of the studied genes. 